\title{
Variational Iteration Method and Adomian Decomposition Method for Fourth-Order Fractional Integro-Differential Equations
}

\author{
S.M.Amer, M. H. Saleh \\ Mathematics Department \\ Faculty of Science \\ Zagazig University, Egypt
}

\author{
M. A. Mohamed \\ Mathematics Department \\ Faculty of Science \\ Suez Canal University, Egypt
}

\author{
N.S.Abdelrhman \\ Mathematics Department \\ Faculty of Science \\ Suez Canal University, \\ Egypt
}

\begin{abstract}
In this paper, linear and nonlinear boundary value problems for fourth-order fractional integro- differential equations are solved by Variational iteration method (VIM) and Adomian decomposition method (ADM). The fractional derivative is considered in the Caputo sense. The solutions of both problems are derived by infinite convergent series. Numerical example are presented to illustrate the efficiency and reliability of two methods.
\end{abstract}

\section{General Terms}

Numerical solutions, Fractional integro-differential equations.

\section{Keywords}

Fractional integro-differential equations, Caputo fractional derivative, Variational iteration method, Adomain decomposition method, Boundary value problems.

\section{INTRODUCTION}

Most fractional differential equation don't have exact analytic solutions, so approximation and numerical techniques must be used . The ADM [ 3,4] is a relatively new approach to provide an analytical approximation to linear and nonlinear problems and it is particularly valuable as a tool for scientists and applied mathematicians. The variational iteration method is based on Lagrange multiplier. These methods have been successfully applied by many author [ 11,16] for finding the analytical approximate solutions as well as numerical approximate solutions of functional equation which arise in scientific and engineering problems. The main feather for the use of VIM is that overcome the difficulties which arise in the ADM during computations of Adomain polynomials [16]. Momani and Aslam Noor [8] established the implementation of ADM to derive analytic approximate solutions of the linear and nonlinear boundary value problems for fourth-order fractional integro-differential equations . the ADM has been used to obtain approximate solutions of a large class of linear and nonlinear differential equations. Recently , the application of the method is extended for fractional differential equations [ 7,9,10]. The purpose of this article is to extend the analysis of VIM and ADM to derive analytic approximate solutions to linear and nonlinear boundary value problems for fourth-order fractional integro-differential equations

$$
\begin{aligned}
D_{* x}^{q} y(x)=f(x)+ & \gamma y(x) \\
+ & \int_{0}^{x}[g(t) y(t)+h(t) f(y(t))] d t, \\
& x \in(0, b), \alpha \in(3,4]
\end{aligned}
$$

with initial conditions

$$
\begin{array}{ll}
y(0)=\gamma_{0}, & \mathrm{y}^{\prime \prime}(0)=\gamma_{2}, \\
y(\mathrm{~b})=\beta_{0}, & \mathrm{y}^{\prime \prime}(0)=\beta_{2},
\end{array}
$$

where $D_{* x} y(x)$ is the $\alpha$ th Caputo fractional derivative of $y(x)$ and $f(y(x))$ is a nonlinear continuous function, $\gamma, \gamma_{0}, \gamma_{2}, \beta_{0}$ and $\beta_{2}$ are real constants and $\mathbf{f}$, $\mathrm{g}$ and $\mathrm{h}$ are given and can be approximated by Taylor polynomials.

\section{BASIC DEFINITIONS}

We give some basic definitions and properties of the fractional calculus theory.

Definition 2.1. A real function $f(x), x>0$, is said to be in the space $C_{\mu}, \mu \in R$, if there exits a real number $p>\mu$, such that $f(x)=x^{p} f_{1}(x)$, where $f_{1}(x) \in C[0, \infty)$.

Definition 2.2. The Riemann-Liouville fractional integral operator of order $\alpha \geq 0$, of a function $\mathbf{f} \in \mathrm{c}_{\mu}, \mu \geq-1$, is defined as

$$
\begin{gathered}
J^{\alpha} f(x)=\frac{1}{\Gamma(\alpha)} \int_{0}^{x}(x-t)^{\alpha-1} f(t) d t, \alpha>0, x>0, \\
J^{0} f(x)=\mathrm{f}(\mathrm{x})
\end{gathered}
$$

The operator $\mathbf{J}^{\boldsymbol{\alpha}}$ satisfies the following properties [1] for $f \in C_{\mu}, \mu \geq-1, \alpha, \beta \geq 0$, and,$\gamma \geq-1$ :

(I) $J^{\alpha} J^{\beta} f(x)=J^{\alpha+\beta} f(x)$.

(II) $J^{\alpha} J^{\beta} f(x)=J^{\beta} J^{\alpha} f(x)$.

(III) $J^{\alpha} x^{\gamma}=\frac{\Gamma(\gamma+1)}{\Gamma(\gamma+1+\alpha)} x^{\alpha+\gamma} ; x>0$. 
Definition 2.3. The fractional derivative of $f(x)$ in the Caputo sense is defined as

$D_{* \mathrm{X}}^{\alpha} f(x)=J^{m-\alpha} D^{m} f(x)$

$$
=\frac{1}{\Gamma(m-\alpha)} \int_{0}^{x}(x-t)^{m-\alpha-1} f^{m}(t) d t,
$$

for $m-1<\alpha \leq m, m \in N, x>0, f \in c_{-1}^{m}$

Also, we need here two of its basic properties

Lemma 2.1. If $m-1<\alpha \leq m, m \in N$ and

$$
\begin{aligned}
& f \in c_{\mu}^{m}, \mu \geq-1 \text {, then } \\
& D_{* x}^{\alpha} J^{\alpha} f(x)=\mathrm{f}(\mathrm{x}), \\
& J^{\alpha} D_{* x}^{\alpha} f(x)=\mathrm{f}(\mathrm{x})-\sum_{k=0}^{m-1} f^{(k)}\left(0^{+}\right) \frac{x^{k}}{k !}, x>0 .
\end{aligned}
$$

\section{VARIATIONAL ITERATION \\ METHOD}

To illustrate the basic concepts of VIM, consider the fractional differential $\mathrm{Eq}(1.1)$ with boundary conditions (1.2) , (1.3) . According to VIM , we can construct the correction functional for $\mathrm{Eq}(1.1)$ as:

$$
\begin{aligned}
& y_{k+1}(x) \\
& =y_{k}(x)+J^{\beta}\left[\lambda \left(D_{* x}^{\alpha} y_{k}(x)-f(x)-\gamma \tilde{y}_{k}(x)\right.\right. \\
& \left.\left.\left.-\int_{0}^{x}\left[g(p) \tilde{y}_{k}(p)+h(p) f\left(\tilde{y}_{k}(p)\right)\right)\right] d p\right)\right] \\
& =y_{k}(x)+\frac{1}{\Gamma(\beta)} \int_{0}^{x}(x-s)^{\beta-1} \lambda(s)\left(D_{* x}^{\alpha} y_{k}(s)-f(s)\right. \\
& -\gamma \tilde{y}_{k}(s)-\int_{0}^{s}\left[g(p) \tilde{y}_{k}(p)\right. \\
& \left.\left.+h(p) f\left(\tilde{y}_{k}(p)\right)\right] d p\right) d s
\end{aligned}
$$

where $J^{\beta}$ is the Riemann - Liouville fractional integral operator of order $\beta=\alpha+1-m, \lambda$ is a general multiplier and $\tilde{y}_{k}$ denotes restricted variation i. e $\delta \tilde{y}_{k}=0$.We make some approximation for the identification of an approximate Lagrange multiplier , so the correctional functional (3.1) can be approximately expressed as:

$$
\begin{aligned}
y_{k+1}(x)=y_{k}(x)+ & \int_{0}^{x} \lambda(s)\left(D_{* s}^{4} y_{k}(s)-f(s)-\gamma \tilde{y}_{k}(s)\right. \\
& -\int_{0}^{s}\left[g(p) \tilde{y}_{k}(p)\right. \\
& \left.\left.+h(p) f\left(\tilde{y}_{k}(p)\right)\right] d p\right) d s
\end{aligned}
$$

Making the above correction functional stationary, we obtain the stationary condition :

$1-\left.\lambda^{\prime \prime \prime}(s)\right|_{s=x}=0,\left.\lambda^{\prime \prime}(s)\right|_{s=x}=0,-\left.\lambda^{\prime}(s)\right|_{s=x}=0$,

$\left.\lambda(s)\right|_{s=x}=0, \lambda^{(i v)}(s)=0$

This gives the following Lagrange multiplier $\lambda(s)=\frac{(s-x)^{3}}{6}$

By substitution of (3.3) into functional (3.1), we obtain the following iteration formula

$$
\begin{aligned}
& y_{k+1}(x) \\
& =y_{k}(x)+\frac{1}{6 \Gamma(\alpha-3)} \int_{0}^{x}(x-s)^{\alpha-4}(s-x)^{3}\left(D_{* s}^{\alpha} y_{k}(s)\right. \\
& -f(s)-\gamma y_{k}(s)-\int_{0}^{s}\left[g(p) y_{k}(p)\right. \\
& \left.\left.+h(p) f\left(y_{k}(p)\right)\right] d p\right) d s \\
& =y_{k}(x)-\frac{(\alpha-3)(\alpha-2)(\alpha-1)}{6 \Gamma(\alpha)} \int_{0}^{x}(x \\
& -s)^{\alpha-1}\left(D_{* s}^{\alpha} y_{k}(s)-f(s)-\gamma y_{k}(s)\right. \\
& -\int_{0}^{s}\left[g(p) y_{k}(p)\right. \\
& \left.\left.+h(p) f\left(y_{k}(p)\right)\right] d p\right) d s
\end{aligned}
$$

This yields the following iteration formula:

$$
\begin{aligned}
& y_{k+1}(x) \\
& =y_{k}(x)-\frac{(\alpha-3)(\alpha-2)(\alpha-1)}{6} J^{\alpha}\left(D_{* x}^{\alpha} y_{k}(x)-f(x)\right. \\
& -\gamma y_{k}(x)-\int_{0}^{x}\left[g(p) y_{k}(p)\right. \\
& \left.\left.+h(p) f\left(y_{k}(p)\right)\right] d p\right)
\end{aligned}
$$

The initial approximation y0 can be chosen by the following way which satisfies initial conditions(1.2):

$$
y_{0}(x)=\gamma_{1} x+\frac{\gamma_{2}}{2} x^{2}+\frac{\gamma_{3}}{6} x^{3}
$$

Where $\gamma_{1}=y^{\prime}(0)$ and $\gamma_{3}=y^{\prime \prime \prime}(0)$ are to be determined by applying suitable boundary conditions(1.3).We can obtain the following first-order approximation by substitution of (3.5) into (3.4):

$$
\begin{aligned}
& y_{1}(x) \\
& =y_{0}(x)-\frac{(\alpha-3)(\alpha-2)(\alpha-1)}{\alpha} J^{\alpha}\left(D_{* x}^{\alpha} y_{0}(x)\right. \\
& -f(x)-\gamma y_{0}(x)-\int\left[g(p) y_{0}(p)\right. \\
& \left.\left.+h(p) f\left(y_{0}(p)\right)\right] d p\right) 0
\end{aligned}
$$

Similarly, we can obtain the higher-order approximations. If Nth-order approximate is enough, then imposing boundary conditions (1.3) in Nth-order approximation yields the following system of equations:

$$
\begin{aligned}
& Y_{N}(b)=\beta_{0} \\
& Y^{\prime \prime}{ }_{N}(b)=\beta_{2}
\end{aligned}
$$

from Eqs.(3.7),(3.8),we can find the unknowns $\gamma_{1}=y^{\prime}(0)$, and $\gamma_{3}=y^{\prime \prime \prime}(0)$. Substituting the constant values of $\gamma_{1}$ and $\gamma_{3}$ in Nth-order approximation results the approximate solution of (1.1),(1.2) and (1.3). 


\section{ADOMIAN DECOMPOSITION METHOD}

We consider the boundary value problem for fourthorder fractional integro-differential equation (1.1) subject to the boundary conditions (1.2),(1.3).In the case of $\alpha$ $=4$, the fractional equation reduces to the classical fourth-order integro-differential equation. Applying the operator $\mathbf{J}^{\boldsymbol{\alpha}}$, the inverse of the operator $D_{* x}^{\alpha}$, to both sides of Eq.(1.1) yields

$$
\begin{aligned}
y(x)=\sum_{j=0}^{3} \gamma_{j} \frac{x^{j}}{j !} & +J^{\alpha}[f(x)+\gamma y(x)] \\
& +J^{\alpha}\left(\int_{0}^{x}[g(t) y(t)\right. \\
& +h(t) f(y(t))] d t) .
\end{aligned}
$$

The Adomian's decomposition method [3,4] suggests the solution $\mathrm{y}(\mathrm{x})$ be decomposed by the infinite series of components

$$
y(x)=\sum_{n=0}^{\infty} y_{n}(x),
$$

and the nonlinear function in Eq.(1.1) is decomposed as follows:

$$
F=\sum_{n=0}^{\infty} A_{n}
$$

where $\mathrm{A}_{\mathrm{n}}$ are so-called the Adomain polynomials. Substitutions the decomposition series (4.2) , (4.3) into both sides of (4.1) gives

$$
\begin{aligned}
\sum_{n=0}^{\infty} y_{n}(x)=\sum_{j=0}^{3} \gamma_{j} & \frac{x^{j}}{j !}+j^{\alpha}\left[f(x)+\gamma \sum_{n=0}^{\infty} y_{n}(x)\right] \\
& +j^{\alpha}\left[\int _ { 0 } ^ { x } \left[g(t) \sum_{n=0}^{\infty} y_{n}(x)\right.\right. \\
& \left.\left.+h(t) \sum_{n=0}^{\infty} A_{n}\right] d t\right]
\end{aligned}
$$

From this equation, the iterates are determined by the following recursive way

$$
\begin{gathered}
y_{0}(x)=\sum_{j=0}^{3} \gamma_{j} \frac{x^{j}}{j !}+J^{\alpha} f(x), \\
y_{n+1}(x)=\gamma J^{\alpha} y_{n}(x)+J^{\alpha}\left[\int_{0}^{x}\left[g(t) y_{n}(t)+h(t) A_{n}\right] d t\right], n \geq \\
0, \quad(4.5)
\end{gathered}
$$

Where $\gamma_{1}=y^{\prime}(0)$ and $\gamma_{3}=y^{\prime \prime \prime}(0)$ are to be determined and the Adomain polynomials $A_{n}$ are given by [4]

$$
A_{n}=\frac{1}{n !}\left[\frac{d^{n}}{d \lambda^{n}} F\left(\sum_{n=0}^{\infty} \lambda^{k} y_{k}\right)\right]_{\lambda=0}
$$

The decomposition series solutions are generally converge very rapidly. The convergence of the decomposition series have investigated by several author $[2,5,6,12,14,15]$. For later numerical computation, let the expression

$$
\phi_{N}(x)=\sum_{n=0}^{N-1} y_{n}(x)
$$

denote the $\mathrm{N}$-term approximation to $y(x)$. Now we determined $\gamma_{1}$ and $\gamma_{3}$ by imposing boundary conditions (1.2),(1.3) on (4.7) gives the system

$$
\begin{gathered}
\phi_{N}(b)=y_{0}(b)+y_{1}(b)+\cdots+y_{N-1}(b)=\beta_{0} \\
\phi^{\prime \prime}{ }_{N}(b)=y^{\prime \prime}{ }_{0}(b)+y^{\prime \prime}{ }_{1}(b)+\cdots+y^{\prime \prime}{ }_{N-1}(b) \\
=\beta_{2},
\end{gathered}
$$

from which the unknowns $\gamma_{1}$ and $\gamma_{3}$ can be found. Once we have the values for $\gamma_{1}$ and $\gamma_{3}$, expression (4.7) serves as an approximate solution of (1.1) , (1.2) and (1.3).

\section{APPLICATIONS AND RESULTS}

We have applied VIM and ADM to fourth-order linear and nonlinear fractional integro-differential equations , we have applied the methods to two different examples with known exact solution for the special case $\alpha=4$.

Example5.1.

Consider the following linear fourth-order Fractional integro-differential equation

$D_{* x}^{\alpha} y(x)=1+(5+x) e^{x}+y(x)-\int_{0}^{x} y(t) d t, 0<x<$

$1,3<\alpha \leq 4$

subject to the boundary conditions

$$
\begin{array}{ll}
y(0)=0 & y^{\prime \prime}(0)=2, \\
y(1)=e & y^{\prime \prime}(1)=3 e,
\end{array}
$$

The exact solution, when, $\alpha=4$,is

$$
y(x)=x e^{x} \text {. }
$$

In order to avoid the difficult fraction integration in two methods, we can take the truncated Taylor expansion for the exponential term as

$$
e^{x} \sim 1+x+\frac{x^{2}}{2 !}+\frac{x^{3}}{3 !}
$$

First, by ADM, the recursive Adomain decomposition algorithm is

$$
\begin{gathered}
y_{0}(x)=A x+x^{2}+\frac{B x^{3}}{3 !}+J^{\alpha}\left[1+5 e^{x}+x e^{x}\right], \\
y_{n+1}(x)=J^{\alpha} y_{n}(x)-J^{\alpha}\left(\int_{0}^{x} y_{n}(t) d t\right), n \geq 0
\end{gathered}
$$

where the constants $A=y^{\prime}(0)$ and $B=y^{\prime \prime \prime}(0)$ are to be determined. Using the recursive algorithm (5.5),yields the 2-term approximation

$$
\begin{aligned}
& \varphi_{2}(x)=A x+x^{2}+\frac{B x^{3}}{6}+\frac{6 x^{\alpha}}{\Gamma(\alpha+1)}+\frac{(6+A) x^{\alpha+1}}{\Gamma(\alpha+2)}+ \\
& \frac{(9-A) x^{\alpha+2}}{\Gamma(\alpha+3)}+\frac{(6+B) x^{\alpha+3}}{\Gamma(\alpha+4)}+\frac{(4-B) x^{\alpha+4}}{\Gamma(\alpha+5)}+\frac{6 x^{2 \alpha}}{\Gamma(2 \alpha+1)}+\frac{x^{2 \alpha+2}}{\Gamma(2 \alpha+3)}+ \\
& \frac{x^{2 \alpha+3}}{\Gamma(2 \alpha+4)}-\frac{4 x^{2 \alpha+4}}{\Gamma(2 \alpha+5)}-\frac{4 x^{2 \alpha+5}}{\Gamma(2 \alpha+6)}
\end{aligned}
$$

Second ,by VIM, the iteration formula can be expressed in the following form:

$$
\begin{aligned}
& y_{k+1}(x)=y_{k}(x)-\frac{(\alpha-3)(\alpha-2)(\alpha-1)}{6} J^{\alpha}\left(D_{* x}^{\alpha} y_{k}(x)-1-\right. \\
& \left.5 e^{x}-x e^{x}-y_{k}(x)+\int_{0}^{x} y_{k}(t) d t\right), n \geq 0
\end{aligned}
$$

and assume that an initial approximation has the following form which satisfies the initial conditions (5.2), (5.3):

$$
y_{0}(x)=A x+x^{2}+\frac{B x^{3}}{3 !}
$$

Now, by iteration formula (5.7) first-order approximation takes the following form

$$
\begin{aligned}
& y_{1}(x)=y_{0}(x)-\frac{(\alpha-3)(\alpha-2)(\alpha-1)}{6} J^{\alpha}\left(D_{* x}^{\alpha} y_{0}(x)-1-5 e^{x}-\right. \\
& \left.x e^{x}-y_{0}(x)+\int_{0}^{x} y_{0}(t) d t\right), \\
& y_{1}(x)=A x+x^{2}+\frac{B x^{3}}{3 !}-\frac{(\alpha-3)(\alpha-2)(\alpha-1)}{6} x^{\alpha} \times\left(\frac{-6}{\Gamma(\alpha+1)}-\right. \\
& \frac{(A+6)}{\Gamma(\alpha+2)} x+\frac{(A-9)}{\Gamma(\alpha+3)} x^{2}-\frac{(B+6)}{\Gamma(\alpha+4)} x^{3}+\frac{(B-4)}{\Gamma(\alpha+5)} x^{4} .
\end{aligned}
$$


Table1. Values of the constants A and B for different values of $\alpha$ by two methods using(5.6),(5.9).

\begin{tabular}{|c|c|c|}
\hline$\alpha$ & \multicolumn{2}{|c|}{ VIM } \\
\hline & $\mathrm{A}$ & $\mathrm{B}$ \\
\hline 3.25 & 0.759452666724677 & 5.07080968251894 \\
\hline 3.5 & 0.868738025237750 & 3.82120800255373 \\
\hline 3.75 & 1.00138785157674 & 2.60083459995043 \\
\hline 4 & 1.13441232626516 & 1.57157467519467 \\
\hline$\alpha$ & & $\mathrm{ADM}$ \\
\hline & & $\mathrm{A}$ \\
\hline 3.25 & 1.26589301045649 & -3.15911802210204 \\
\hline 3.5 & 1.24354885848781 & -1.26316162748865 \\
\hline 3.75 & 1.20912540791374 & 0.216519469700978 \\
\hline 4 & 1.13566559250585 & 1.56311706233720 \\
\hline & & \\
\hline
\end{tabular}

Table 2. Approximate solution of (5.1) for different values of $\alpha$ by the two methods.

\begin{tabular}{|c|c|l|l|l|l|}
\hline $\mathrm{x}$ & \multicolumn{1}{|c|}{ Exact } & $\begin{array}{r}\text { ADM } \\
\alpha=3.25\end{array}$ & $\begin{array}{c}\text { ADM } \\
\alpha=3.5\end{array}$ & $\begin{array}{c}\text { ADM } \\
\alpha=3.75\end{array}$ & $\begin{array}{c}\text { ADM } \\
\alpha=4\end{array}$ \\
\hline 0.1 & 0.1105171 & 0.1364819 & 0.1343119 & 0.1310146 & 0.1238527 \\
\hline 0.2 & 0.2442806 & 0.2930708 & 0.2889740 & 0.2830248 & 0.2696370 \\
\hline 0.3 & 0.4049576 & 0.4713378 & 0.4656599 & 0.4579892 & 0.4399115 \\
\hline 0.4 & 0.5967299 & 0.6740786 & 0.6672492 & 0.6588760 & 0.6379957 \\
\hline 0.5 & 0.8243606 & 0.9052706 & 0.8978026 & 0.8897026 & 0.8680642 \\
\hline 0.6 & 1.0932713 & 1.1701176 & 1.1626087 & 1.1556119 & 1.1352506 \\
\hline 0.7 & 1.4096269 & 1.4751311 & 1.4682648 & 1.4629695 & 1.4457639 \\
\hline 0.8 & 1.7804327 & 1.8282372 & 1.8227817 & 1.8194796 & 1.8070167 \\
\hline 0.9 & 2.2136428 & 2.2389029 & 2.2357093 & 2.2343179 & 2.2277683 \\
\hline 1 & 2.7182818 & 2.7182818 & 2.7182818 & 2.7182819 & 2.7182818 \\
\hline
\end{tabular}

\begin{tabular}{|c|c|c|c|c|c|}
\hline $\mathrm{x}$ & Exact & $\begin{array}{r}\text { VIM } \\
\alpha=3.25\end{array}$ & $\begin{array}{l}\text { VIM } \\
\alpha=3.5\end{array}$ & $\begin{array}{l}\text { VIM } \\
\alpha=3.75\end{array}$ & $\begin{array}{l}\text { VIM } \\
\alpha=4\end{array}$ \\
\hline 0.1 & 0.1105171 & 0.0868394 & 0.0975630 & 0.1106119 & 0.1237288 \\
\hline 0.2 & 0.2442806 & 0.1991309 & 0.2194499 & 0.2442926 & 0.2693976 \\
\hline 0.3 & 0.4049576 & 0.3424951 & 0.3703951 & 0.4046883 & 0.4395736 \\
\hline 0.4 & 0.5967299 & 0.5226934 & 0.5555049 & 0.5960490 & 0.6375844 \\
\hline 0.5 & 0.8243606 & 0.7456229 & 0.7802510 & 0.8232521 & 0.8676129 \\
\hline 0.6 & 1.0932713 & 1.0173234 & 1.0504844 & 1.0918480 & 1.1347999 \\
\hline 0.7 & 1.4096269 & 1.3439874 & 1.3724624 & 1.4081181 & 1.4453601 \\
\hline 0.8 & 1.7804327 & 1.7319739 & 1.7528824 & 1.7791444 & 1.8067082 \\
\hline 0.9 & 2.2136428 & 2.1878247 & 2.1989212 & 2.2128888 & 2.2275992 \\
\hline 1 & 2.7182818 & 2.7182818 & 2.7182818 & 2.7182818 & 2.7182818 \\
\hline
\end{tabular}


Fig.1. Comparison of approximate solutions obtained by ADM and VIM with exact solution when $\alpha=3.25$ of example 5.1.

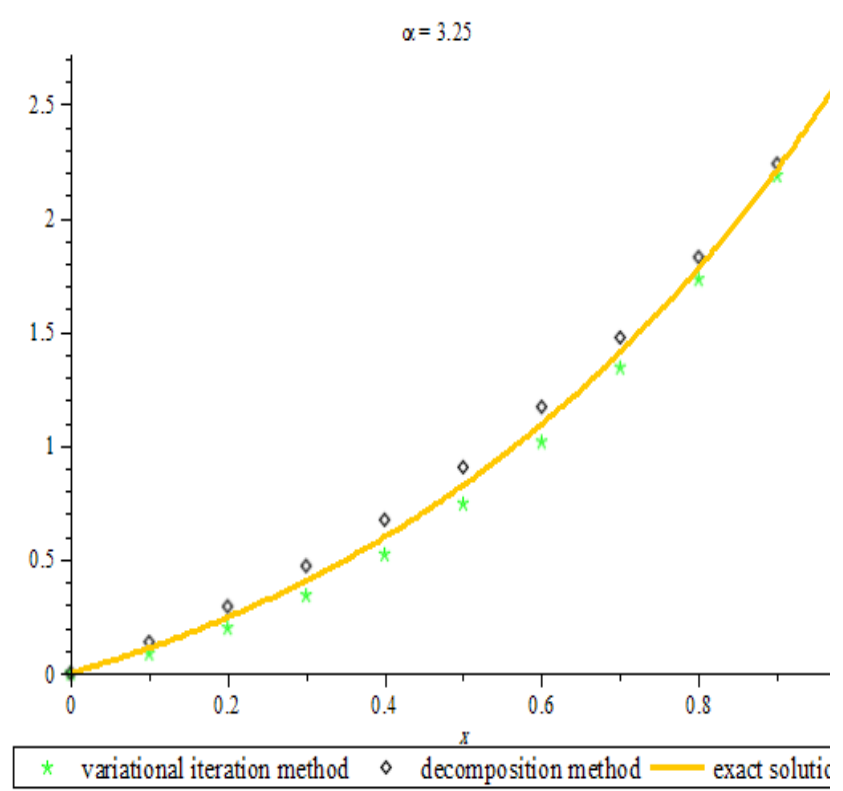

Fig.2. Comparison of approximate solutions obtained by ADM and VIM with exact solution when $\alpha=3.5$ of example 5.1.

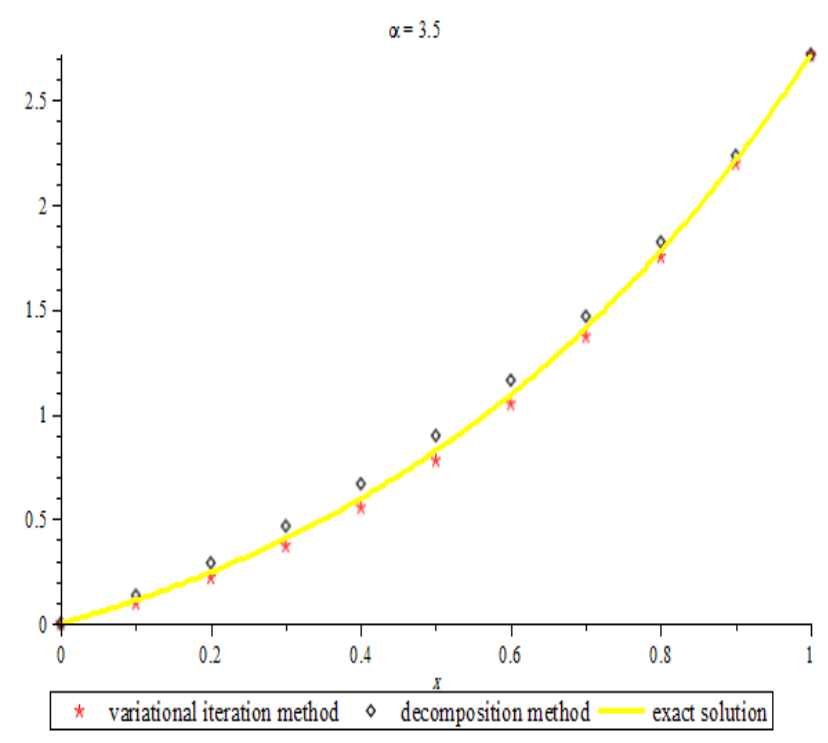

Fig.3. Comparison of approximate solutions obtained by ADM and VIM with exact solution when $\alpha=3.75$ of example 5.1.

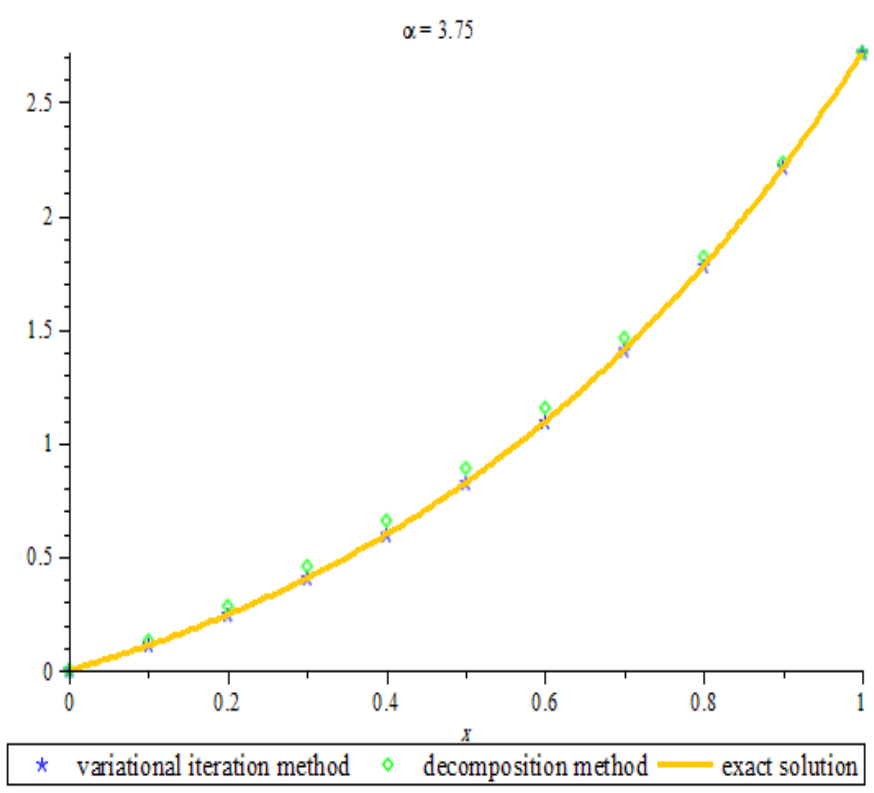

Fig.4. Comparison of approximate solutions obtained by ADM and VIM with exact solution when $\alpha=4$ of example 5.1.

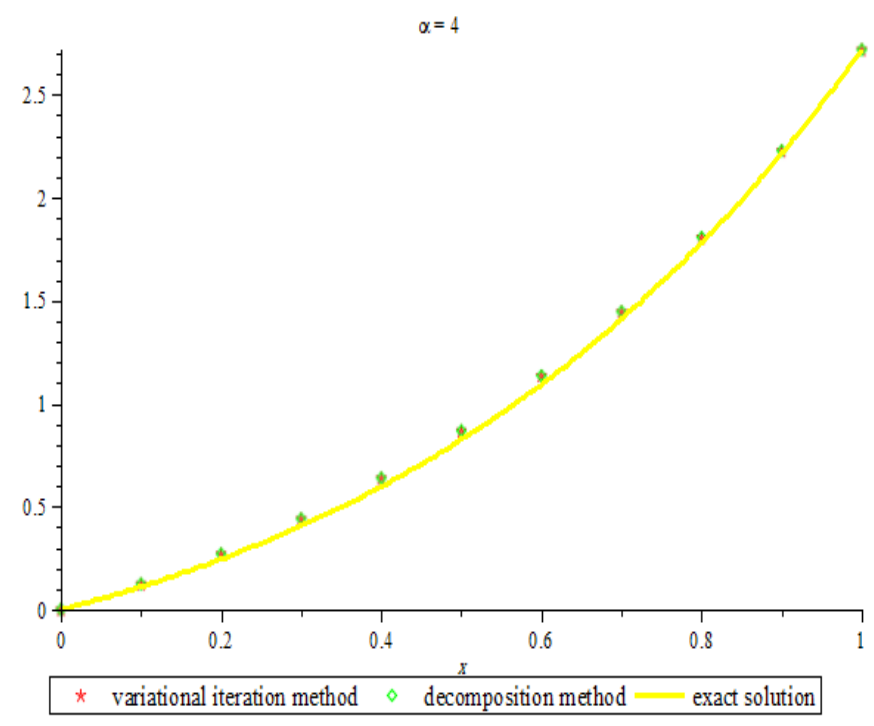

\section{Example5.2.}

Consider the following nonlinear fourth-order Fractional integro-differential equation

$D_{* x}^{\alpha} y(x)=-2 x+e^{-x}+\int_{0}^{x} e^{-t} y^{2}(t) d t, 0<x<1,3<$

$\alpha \leq 4$

subject to the boundary conditions
$y(0)=2$
$y^{\prime \prime}(0)=1$,
$y(1)=e+1 \quad y^{\prime \prime} 1=e$,

The exact solution, when, $\alpha=4$, is

$y(x)=e^{x}+1$ 
First, by ADM, the recursive Adomain decomposition algorithm is

$y_{0}(x)=2+A x+\frac{x^{2}}{2}+\frac{B x^{3}}{3 !}+J^{\alpha}\left[-2 x+e^{-x}\right]$,

$y_{n+1}(x)=J^{\alpha}\left(\int_{0}^{x} e^{-t} A_{n}(t) d t\right), n \geq 0$

where the constants $A=y^{\prime}(0)$ and $B=y^{\prime \prime \prime}(0)$ are to be determined. Using the recursive algorithm (5.14),(4.6) to calculate the Adomain polynomials for the nonlinear term $f(y)=y^{2}$, we obtain the 2-term approximation $\phi_{2}(x)=y_{0}(x)+J^{\alpha}\left(\int_{0}^{x}\left(1-t+\frac{t^{2}}{2}-\frac{t^{3}}{6}\right) A_{0}(t) d t\right), \quad$ (5.15) Where $y_{0}(x)=2+A x+\frac{x^{2}}{2}+\frac{B x^{3}}{6}+\frac{x^{\alpha}}{\Gamma(\alpha+1)}-\frac{3 x^{\alpha+1}}{\Gamma(\alpha+2)}+$ $\frac{x^{\alpha+2}}{\Gamma(\alpha+3)^{\prime}}$

$A_{0}(x)=\left(y_{0}(x)\right)^{2}$

Second ,by VIM, the iteration formula can be expressed in the following form:

$y_{k+1}(x)=y_{k}(x)-\frac{(\alpha-3)(\alpha-2)(\alpha-1)}{6} J^{\alpha}\left(D_{* x}^{\alpha} y_{k}(x)+2 x-\right.$ $\left.e^{-x}-\int_{0}^{x} e^{-t} y_{k}^{\alpha}(t) d t\right)$, (5.16)

and assume that an initial approximation has the following form which satisfies the initial conditions (5.11),(5.12):

$y_{0}(x)=2+A x+\frac{x^{2}}{2}+\frac{B x^{3}}{3 !}$

Now, by iteration formula (5.16) first-order approximation takes the following form $y_{1}(x)=y_{0}(x)-\frac{(\alpha-3)(\alpha-2)(\alpha-1)}{6} J^{\alpha}\left(D_{* x}^{\alpha} y_{0}(x)+2 x-e^{-x}-\right.$ $\left.\int_{0}^{x} e^{-t} y_{0}(t) d t\right)$

Table 3. Values of the constants A and B for different values of $\alpha$ by two methods using(5.15),(5.18)

\begin{tabular}{|c|c|c|}
\hline$\alpha$ & \multicolumn{2}{|c|}{ VIM } \\
\hline & $\mathrm{A}$ & B \\
\hline 3.25 & 0.9411915962 & 1.554002072 \\
\hline 3.5 & 0.9576121209 & 1.360417112 \\
\hline 3.75 & 0.9782883390 & 1.168049074 \\
\hline 4 & 0.9994797370 & 1.003434856 \\
\hline$\alpha$ & \multicolumn{2}{|c|}{$\mathrm{ADM}$} \\
\hline & $\mathrm{A}$ & B \\
\hline 3.25 & 1.016397286 & 0.2735113225 \\
\hline 3.5 & 1.018047562 & 0.5430999829 \\
\hline 3.75 & 1.011417998 & 0.7858792769 \\
\hline 4 & 1.000318280 & 0.9971537827 \\
\hline
\end{tabular}

Table 4. Approximate solution of (5.10) for different values of $\alpha$ by the two methods.

\begin{tabular}{|c|c|c|c|c|c|}
\hline $\mathrm{x}$ & Exact & $\begin{array}{r}\text { ADM } \\
\alpha=3.25\end{array}$ & $\begin{array}{r}\text { ADM } \\
\alpha=3.5\end{array}$ & $\begin{array}{c}\text { ADM } \\
\alpha=3.75\end{array}$ & $\begin{array}{l}\text { ADM } \\
\alpha=4\end{array}$ \\
\hline 0.1 & 2.105170918 & 2.1067548 & 2.1069231 & 2.1062837 & 2.1052023 \\
\hline
\end{tabular}

\begin{tabular}{|c|c|c|c|c|c|}
\hline X & Exact & $\begin{array}{c}\text { VIM } \\
\alpha=3.25\end{array}$ & $\begin{array}{c}\text { VIM } \\
\alpha=3.5\end{array}$ & $\begin{array}{c}\text { VIM } \\
\alpha=3.75\end{array}$ & $\begin{array}{c}\text { VIM } \\
\alpha=4\end{array}$ \\
\hline 0.1 & 2.105170918 & 2.0993863 & 2.1009966 & 2.1030301 & 2.1051195 \\
\hline 0.2 & 2.221402758 & 2.2103897 & 2.2134368 & 2.2173056 & 2.2213033 \\
\hline 0.3 & 2.349858808 & 2.3346540 & 2.3388306 & 2.3441660 & 2.3497182 \\
\hline 0.4 & 2.491824698 & 2.4738447 & 2.4787465 & 2.4850470 & 2.4916532 \\
\hline 0.5 & 2.648721271 & 2.6296470 & 2.6348088 & 2.6414825 & 2.6485325 \\
\hline 0.6 & 2.822118800 & 2.8037663 & 2.8086983 & 2.8151092 & 2.8219295 \\
\hline 0.7 & 3.013752707 & 2.9979289 & 3.0021549 & 3.0076737 & 3.0135822 \\
\hline 0.8 & 3.225540928 & 3.2138834 & 3.2169804 & 3.2210403 & 3.2254099 \\
\hline 0.9 & 3.459603111 & 3.4534019 & 3.4550432 & 3.4572004 & 3.4595309 \\
\hline 1 & 3.718281828 & 3.7182818 & 3.7182818 & 3.7182818 & 3.7182818 \\
\hline 0.2 & 2.221402758 & 2.2243216 & 2.2246555 & 2.2234820 & 2.2214626 \\
\hline 0.3 & 2.349858808 & 2.3537433 & 2.3542197 & 2.3526659 & 2.3499415 \\
\hline 0.4 & 2.491824698 & 2.4962500 & 2.4968280 & 2.4950673 & 2.4919220 \\
\hline 0.5 & 2.648721271 & 2.6532486 & 2.6538748 & 2.6520830 & 2.6488226 \\
\hline 0.6 & 2.822118800 & 2.8263283 & 2.8269419 & 2.8252841 & 2.8222124 \\
\hline 0.7 & 3.013752707 & 3.0172708 & 3.0178087 & 3.0164291 & 3.0138273 \\
\hline 0.8 & 3.225540928 & 3.2280644 & 3.2284669 & 3.2274805 & 3.2255883 \\
\hline 0.9 & 3.459603111 & 3.4609192 & 3.4611360 & 3.4606227 & 3.4596219 \\
\hline 1 & 3.718281828 & 3.7182818 & 3.7182818 & 3.7182818 & 3.7182818 \\
\hline
\end{tabular}


Fig.5. Comparison of approximate solutions obtained by ADM and VIM with exact solution when $\alpha=3.25$ of example 5.2

$$
\alpha=3.25
$$

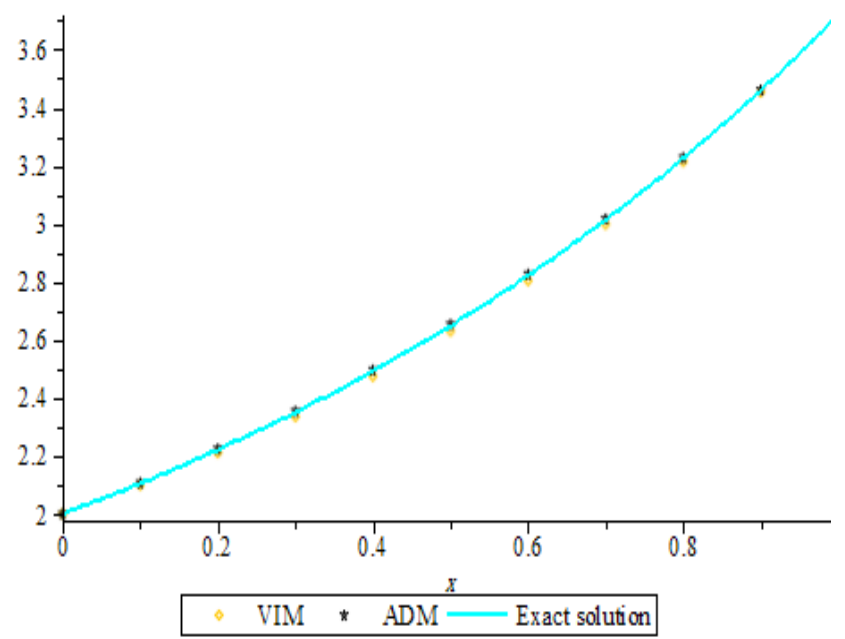

Fig.5. Comparison of approximate solutions obtained by ADM and VIM with exact solution when $\alpha=3.5$ of example 5.2

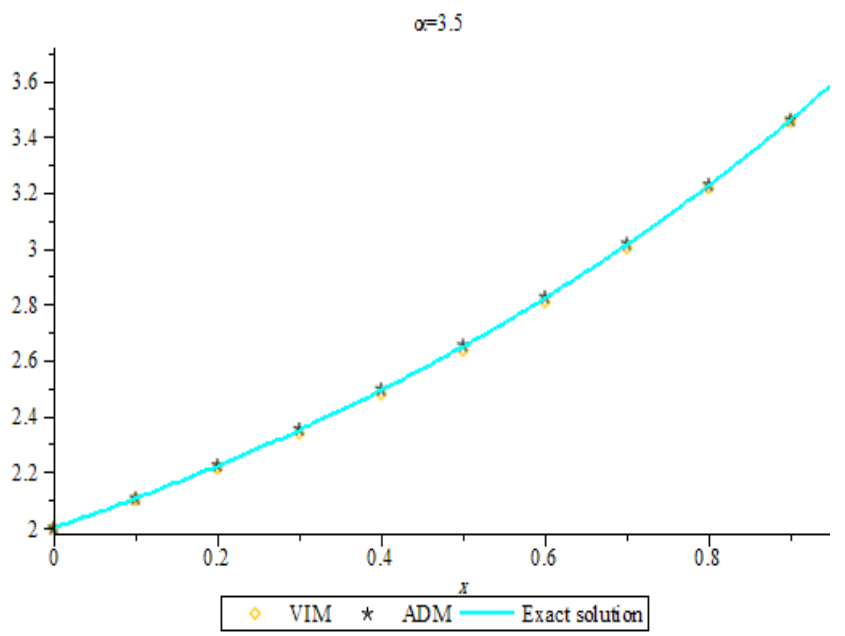

Fig.5. Comparison of approximate solutions obtained by ADM and VIM with exact solution when $\alpha=3.75$ of example 5.2

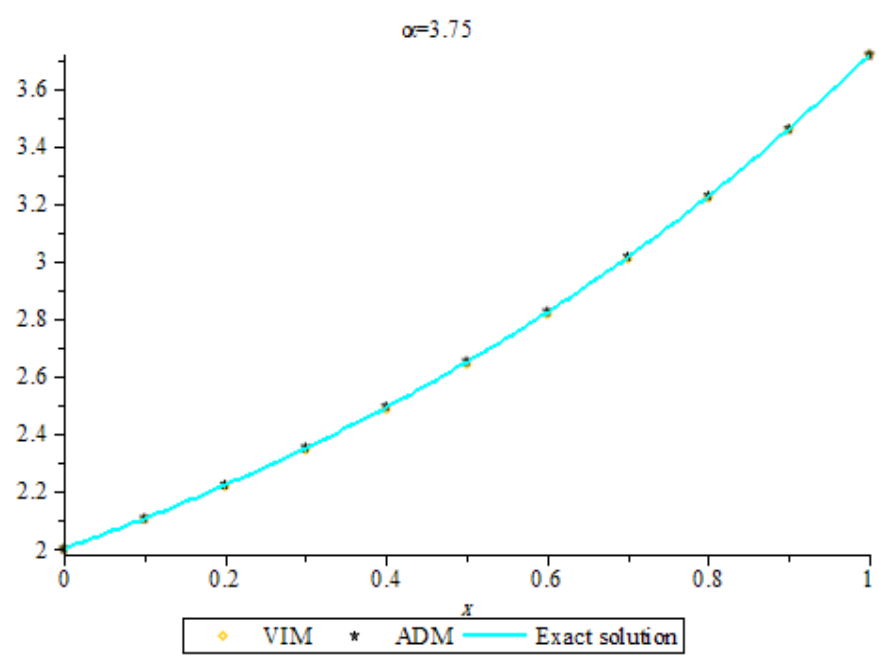

Fig.5. Comparison of approximate solutions obtained by ADM and VIM with exact solution when $\alpha=4$ of example 5.2

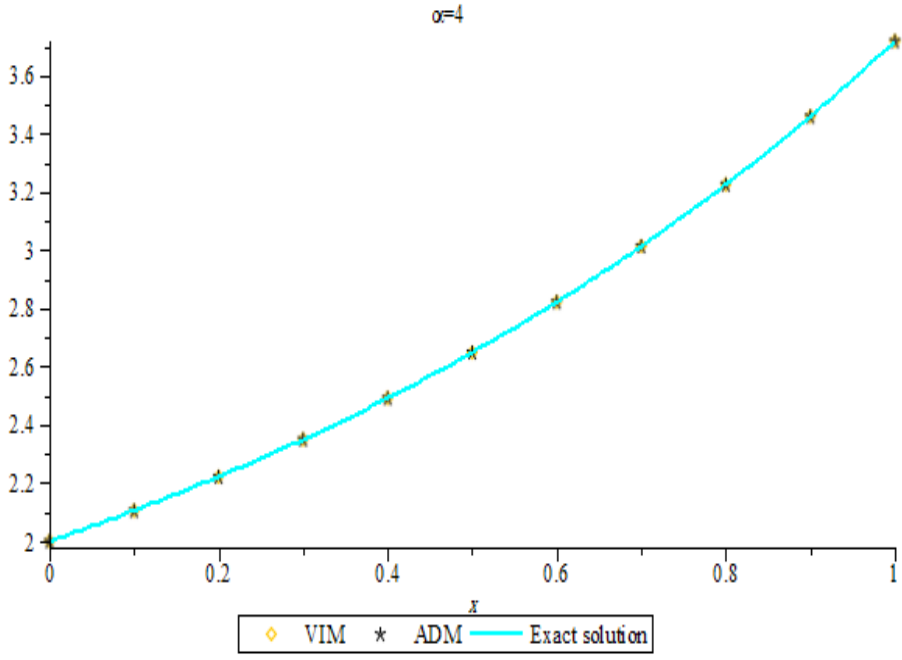

\section{CONCLUSION}

In this paper, we have applied the Variational iteration and Adomain decomposition methods for finding the solution of linear and nonlinear boundary value problem for fourth-order fractional integro-differential equations. The integro-differential equations converted to infinite convergent series by two methods .Comparisons of VIM and ADM with exact solution have been shown by graphs which show the efficiency of the methods and we find that VIM results better than ADM.

\section{ACKNOWLEDGMENTS}

Our thanks to the experts who have contributed towards development of the template. 


\section{REFERENCES}

[1] AY. Luchko , R . Groreflo, The initial value problem for some fractional equations with the Caputo derivative , Preprint series A08-98, Fachbreich mathmatik und informatik, Freic Universitat Berlin , 1998.

[2] A. Repaci , Nonlinear Dynamical Systems : On the Accuracy of Adomain's Decomposition Method , Appl.Mth.lett 3(3)(1990) 35-39.

[3] G.Adomain ,Areview of the Decomposition Method in Applied Mathmatics, J.Math.Anal.Appl. 135(1988) 501544.

[4] G.Adomain ,Solving Frontier Problems of Physics : The Decomposition Method, Kluwer Aca- demic Publishers,Boston ,1994.

[5] K.Abbaoui,Y.Cherruault,Convergence of Adomain's Method Applied to Differential Equations, Comput.Math.Appl.28(5)(1996) 103-109.

[6] K.Abbaoui,Y.Cherruault,New ideas for Proving Convergence of Decomposition Methods, Comput.Math.Appl.29(7)(1996) 103-108.

[7] N.T.Shawagfeh,Analytical Approximate Solutions for Nonlinear Fractional Differential Equa- tions , Appl.Math.Comput.123(2001) 133-140.
[8] S.Momani,M.Aslam Noor, Numerical Methods for Fourth Order Fractional Integro-Differential Equations , Applied Mathematics and Computations 182 (2006) 754 - 760. .

[9] S.Momani,K.Al-Khaled, Numerical Solutions for Systems of Fractional Differential Equations by the Decomposition Method Appl.Math.Comput.162(3)(2005) 1351-1365 .

[10] S.Momani,Numerical Simulation of Adynamic System Containing Fractional Derivatives, Int.J.Numer.Simulat,in press .

[11] V.Daftardar-Gejji,Hossein Jafari , Solving Amulti-Order Fractional Differential Equation Using Adomain Decomposition , Applied Mathmatics and Computation 189 (2007) 541-548

[12] V.Seng , K.Abbaoui , Adomain's Polynomials for Nonlinear Operators , Math . Comput . Modell. 24(1)(1996) 59-65.

[13] Yasir Nawaz ,Variational Iteration Method and Homotopy Perturbation Method for Fourth- Order Fractional Integro-Differential Equations , Comput and Mathmatics .61 (2011) 2330-2341.

[14] Y.Cherruault , convergence of Adomain's Method , Kybernetes 18(1989) 31-38. 